\title{
PEARL: A Non-interventional Study of Real-World Alirocumab Use in German Clinical Practice
}

\author{
Klaus G. Parhofer ${ }^{1} \cdot$ Berndt von Stritzky ${ }^{2} \cdot$ Nicole Pietschmann ${ }^{2} \cdot$ Cornelia Dorn $^{2} \cdot$ W. Dieter Paar ${ }^{2}$
}

Published online: 6 July 2019

(c) The Author(s) 2019

\begin{abstract}
Background Several lipid guidelines recommend that proprotein convertase subtilisin/kexin type 9 inhibitors should be considered for patients with atherosclerotic cardiovascular disease who are inadequately treated with maximally tolerated lipid-lowering treatment.

Objectives The PEARL study assessed the efficacy and safety of the proprotein convertase subtilisin/kexin type 9 inhibitor alirocumab in patients with hypercholesterolemia in a real-world setting.

Methods PEARL was an open, prospective, multicenter, non-interventional study conducted in Germany. Patients $(n=619)$ for whom treating physicians decided to use alirocumab 75 or $150 \mathrm{mg}$ every 2 weeks according to German guidelines (lowdensity lipoprotein cholesterol $>1.8 / 2.6 \mathrm{mmol} / \mathrm{L}[>70 / 100 \mathrm{mg} / \mathrm{dL}]$, depending on cardiovascular risk, despite maximally tolerated statin therapy with/without other non-alirocumab lipid-lowering therapy) were enrolled and followed for 24 weeks. Physicians could adjust the alirocumab dose based on their clinical judgment. The primary efficacy endpoint was low-density lipoprotein cholesterol reduction from baseline (prior to alirocumab therapy) to week 24.

Results Overall, $72.8 \%$ of patients reported complete or partial statin intolerance. Mean low-density lipoprotein cholesterol was $4.7 \mathrm{mmol} / \mathrm{L}(180.5 \mathrm{mg} / \mathrm{dL})$ and $2.3 \mathrm{mmol} / \mathrm{L}(89.8 \mathrm{mg} / \mathrm{dL})$ at baseline and week 24 , respectively. Least-squares mean percentage change from baseline to week 24 in low-density lipoprotein cholesterol was $-48.6 \%$. Initial alirocumab dose was $75 \mathrm{mg}$ in $72.9 \%$ of patients and $150 \mathrm{mg}$ in $24.5 \%$ of patients; $19.6 \%$ of patients received an alirocumab dose increase (75 to $150 \mathrm{mg}$ ) and $1.6 \%$ of patients received a dose decrease. Adverse events were reported in $10.3 \%$ of patients, with myalgia being the most common. Conclusions In a real-world setting in Germany, alirocumab was used in patients who had high baseline low-density lipoprotein cholesterol levels with/without statin intolerance. Efficacy and safety were consistent with findings observed in the ODYSSEY Phase III program.
\end{abstract}

\section{Key Points}

The 24-week PEARL study assessed the lipid-lowering efficacy and safety of alirocumab, a proprotein convertase subtilisin/kexin type 9 inhibitor, in patients at cardiovascular risk despite their previous lipid-lowering therapies in a real-world setting in Germany

In total, $72.8 \%$ of patients reported complete or partial statin intolerance and mean low-density lipoprotein choles-

Electronic supplementary material The online version of this article (https://doi.org/10.1007/s40801-019-0158-0) contains supplementary material, which is available to authorized users.

Klaus G. Parhofer

Klaus.Parhofer@med.uni-muenchen.de

1 Medical Department IV-Grosshadern, University of Munich, Marchioninistr. 15, 81377 Munich, Germany

2 Medical Department, Sanofi-Aventis Deutschland GmbH, Berlin, Germany terol was $4.7 \mathrm{mmol} / \mathrm{L}(180.5 \mathrm{mg} / \mathrm{dL})$ at baseline; enrolled patients received alirocumab dosing of $75 \mathrm{mg}$ or $150 \mathrm{mg}$ every 2 weeks based on physicians' clinical judgment

In alirocumab-treated patients, low-density lipoprotein cholesterol was reduced from baseline by $48.6 \%$ at week 24 , including $43.6 \%$ of patients remaining on alirocumab $75 \mathrm{mg}$ every 2 weeks throughout; alirocumab was generally well tolerated 


\section{Introduction}

Patients with elevated low-density lipoprotein cholesterol (LDL-C) levels have an increased risk of atherosclerotic cardiovascular disease events [1]. The European Society of Cardiology/European Atherosclerosis Society Task Force and other consensus statements and lipid guidelines indicate that a proprotein convertase subtilisin/kexin type 9 (PCSK9) inhibitor should be considered for reducing LDL-C in patients with atherosclerotic cardiovascular disease and/or diabetes mellitus (DM) who are not adequately treated with maximally tolerated statins and/or ezetimibe [2-6].

Alirocumab, a fully human monoclonal antibody to PCSK9, is approved in the European Union and USA as an adjunct to diet on maximally tolerated statin therapy [7, 8]. Alirocumab significantly reduced LDL-C levels vs. control (ezetimibe or placebo) in ODYSSEY Phase III studies including patients with and without heterozygous familial hypercholesterolemia ( $\mathrm{HeFH})$, either as monotherapy or in addition to statin \pm other lipid-lowering therapies (LLTs) [9-14]. Recently, the results of the ODYSSEY OUTCOMES study (NCT01663402) in 18,924 patients with post-acute coronary syndrome (ACS) randomized 1-12 months after ACS showed that alirocumab therapy translates into a significant cardiovascular risk reduction. Alirocumab reduced major adverse cardiac events (defined as coronary heart disease [CHD] death, non-fatal myocardial infarction, fatal and non-fatal ischemic stroke, or unstable angina requiring hospitalization) by $15 \%$ (absolute risk reduction: $1.6 \%$ ) and was associated with a reduction in all-cause death by $15 \%$ (absolute risk reduction: $0.6 \%$ ) compared with placebo [15]. In patients with atherosclerotic cardiovascular disease and LDL-C levels $\geq 1.8 \mathrm{mmol} / \mathrm{L}$ ( $\geq 70 \mathrm{mg} / \mathrm{dL}$ ), adding the PCSK9 inhibitor evolocumab to statin therapy significantly reduced the risk of cardiovascular events, with a $15 \%$ reduction in the risk of cardiovascular death, myocardial infarction, stroke, hospitalization for unstable angina, or coronary revascularization [16].

In Germany, alirocumab has been reimbursed in patients with $\mathrm{HeFH}$, non-familial hypercholesterolemia $(\mathrm{FH})$, or mixed dyslipidemia with confirmed vascular disease (CHD, cerebrovascular manifestation, or peripheral arterial disease) and other risk factors (e.g., DM) since May 2016 as an adjunct to diet, either in combination with statins and/or other LLTs, who demonstrate persistently high LDL-C levels despite receiving documented maximum dietary therapy and LLTs for 12 months, and who would therefore be indicated for lipid apheresis therapy [17]. In Germany, alirocumab therapy can only be initiated and monitored by specialists (e.g., cardiologists, nephrologists, diabetologists, and endocrinologists) [17].
In a real-life setting, data on efficacy and safety of alirocumab are limited [18-20]. In an overall cohort comprising 238 patients who received either evolocumab or alirocumab at an outpatient clinic in the Netherlands, the mean LDL-C reduction was $58.3 \%$ [18]. In a real-world setting in Germany (527 enrolled patients), alirocumab $150 \mathrm{mg}$ every 2 weeks (Q2W), alirocumab 75 mg Q2W (with possible dose adjustment to $150 \mathrm{mg}$ Q2W), and evolocumab $140 \mathrm{mg}$ Q2W resulted in LDL-C $\leq 1.8 \mathrm{mmol} / \mathrm{L}(\leq 70 \mathrm{mg} / \mathrm{dL})$ after 4 weeks of treatment, by reducing LDL-C levels by $2.4 \mathrm{mmol} / \mathrm{L}$ (94.0 mg/dL), $1.7 \mathrm{mmol} / \mathrm{L}(64.2 \mathrm{mg} / \mathrm{dL})$, and $2.4 \mathrm{mmol} / \mathrm{L}(91.8 \mathrm{mg} / \mathrm{dL})$, respectively [19]. In patients $(n=72)$ who were either taking alirocumab $(57 \%)$ or evolocumab $(43 \%)$ in a university-based outpatient clinic, LDL-C levels were reduced by $55 \%$ after 4 weeks of treatment [20]. The PEARL (Prospektive Nicht-Interventionelle Studie zur Erfassung der WirksAmkeit und VeRträgLichkeit des PCSK9-Inhibitors PRALUENT) study assessed the efficacy and safety of alirocumab in patients with hypercholesterolemia in a real-world setting in Germany for 24 weeks.

\section{Methods}

PEARL was an open-label, prospective, multicenter, noninterventional study conducted according to the German Drug Law ( $\$ 4$ part 23) at 345 centers in Germany (Nichtinterventionelle Studie number: 320) [21]. The decision to prescribe alirocumab had to be independent from study participation and all treatment decisions remained at the discretion of the treating physician. The participating practices were distributed evenly across Germany to allow for a broad geographical representation. The study was performed in accordance with the ethical principles that have their origin in the Declaration of Helsinki, and all applicable amendments laid down by the World Medical Assemblies and the International Conference Harmonization guidelines for Good Clinical Practice. The study protocol was approved by the local institutional review board and independent ethics committee at each site. All patients provided written informed consent prior to documentation.

\subsection{Patients and Study Design}

Patients who were eligible for the study had elevated LDL-C $\geq 1.8 \mathrm{~mol} / \mathrm{L}$ ( $\geq 70 \mathrm{mg} / \mathrm{dL}$; for patients with established cardiovascular disease) or $\geq 2.6 \mathrm{mmol} / \mathrm{L}$ ( $\geq 100 \mathrm{mg} / \mathrm{dL}$; for patients without established cardiovascular disease) despite maximally tolerated non-alirocumab LLT, and their treating physicians decided to use alirocumab according to the summary of product characteristics [8]. Participating patients received alirocumab 75 or $150 \mathrm{mg} \mathrm{Q} 2 \mathrm{~W}$ and were followed for approximately 24 weeks. Study data were 
derived from paper-based case report forms. Physicians could adjust the alirocumab dose (75 or $150 \mathrm{mg} \mathrm{Q2W}$ ) and non-alirocumab LLT based on their own clinical judgment throughout the study.

For this study, statin intolerance was categorized as total or partial statin intolerance. Briefly, total statin intolerance was defined as an inability reported in the past medical history to tolerate two or more statins (one statin at the lowest daily starting dose [i.e., rosuvastatin $5 \mathrm{mg}$, atorvastatin $10 \mathrm{mg}$, simvastatin $10 \mathrm{mg}$, lovastatin $20 \mathrm{mg}$, pravastatin $40 \mathrm{mg}$, fluvastatin $40 \mathrm{mg}$, or pitavastatin $2 \mathrm{mg}$, and another statin at any dose) owing to unexplained skeletal musclerelated symptoms, other than as a result of strain or trauma, that began or increased during statin treatment and resolved with statin discontinuation. Partial statin intolerance was defined as an inability reported in the past medical history to tolerate a sufficient statin dose to reach an individual treatment target, resulting in patients receiving a reduced statin dosage.

For the LLT status before the start of alirocumab therapy, the patient questionnaire was unclear for some physicians, resulting in the reporting of any LLTs taken by the patient in the past and not only the current treatment given at the start of this study. As this resulted in inconsistencies specifically and exclusively in the respective data, LLT status is not reported in this study.

Further details on the definition on FH are presented in the Electronic Supplementary Material (ESM). The study inclusion and exclusion criteria are presented in Table 1 of the ESM.

\subsection{Endpoints and Laboratory Assessments}

The primary efficacy endpoint was the percentage reduction in LDL-C from baseline (prior to start of alirocumab therapy) to week 24 , analyzed with the use of the intention-to-treat (ITT) approach, which included all patients who received at least one dose of alirocumab within this study and who had at least one post-baseline value. Secondary efficacy endpoints included absolute change in LDL-C from baseline to week 24; percentage change from baseline to week 24 in total cholesterol, high-density lipoprotein cholesterol (HDL-C), apolipoprotein B, and triglycerides (TGs; ITT analysis); and the proportion of patients achieving $\mathrm{LDL}-\mathrm{C}<1.8 \mathrm{mmol} / \mathrm{L}(<70 \mathrm{mg} / \mathrm{dL})$ or $<2.6 \mathrm{mmol} / \mathrm{L}$ $(<100 \mathrm{mg} / \mathrm{dL})$, depending on cardiovascular risk. Physicians provided lipid levels by completing the patient questionnaire; no information on the type of lipid measurement was collected. An additional analysis of glycated hemoglobin $\left(\mathrm{HbA}_{1 \mathrm{c}}\right.$; absolute change) was performed for patients with DM.

Patient data were recorded at the start of study drug therapy (baseline [visit 1]; week 0) and after approximately
Table 1 Patient characteristics and lipids at baseline (intention-totreat analysis)

\begin{tabular}{ll}
\hline Characteristics & Patients $(n=612)$ \\
\hline Age, mean (SD), years & $61.6(11.1)$ \\
Male, $n(\%)$ & $394(64.4)$ \\
FH, $n(\%)$ & $311(50.8)$ \\
Hypertension, $n(\%)$ & $423(69.1)$ \\
CHF, $n(\%)$ & $82(13.4)$ \\
DM, $n(\%)$ & $169(27.6)$ \\
Type 1 & $10 / 169(5.9)$ \\
Type 2 & $154 / 169(91.1)$ \\
Insulin dependency & $56 / 169(33.1)$ \\
Any cardiovascular disease, $n$ & $552(90.2)$ \\
(\%) & $443(72.4)$ \\
CHD & $202(33.0)$ \\
ACS & $81(13.2)$ \\
PAD & $56(9.2)$ \\
Cerebrovascular disease & $277(45.3)$ \\
Total statin intolerance, ${ }^{a} n(\%)$ & $169(27.6)$ \\
Partial statin intolerance, ${ }^{b} n(\%)$ & $50(8.2)$ \\
Apheresis, $n(\%)$ & $4.7(1.6)[180.5(60.7)]$ \\
Lipids, mean (SD), mmol/L [mg/dL] & \\
LDL-C & $5.5(1.9)[213.9(72.2)]$ \\
Non-HDL-C & $1.3(0.5)[50.8(19.7)]$ \\
HDL-C & $6.9(2.0)[266.6(76.1)]$ \\
Total cholesterol & $2.9(4.3)[256.8(377.9)]$ \\
TGs & $2.0(1.5: 3.1)[178.0(129.0: 268.0)]$ \\
$\quad$ Median (Q1:Q3) & \\
\hline &
\end{tabular}

$A C S$ acute coronary syndrome, $C H F$ congestive heart failure, $C H D$ coronary heart disease, $D M$ diabetes mellitus, $F H$ familial hypercholesterolemia, $H D L-C$ high-density lipoprotein cholesterol, $L D L-C$ low-density lipoprotein cholesterol, $P A D$ peripheral arterial disease, $S D$ standard deviation, $T G$ triglyceride

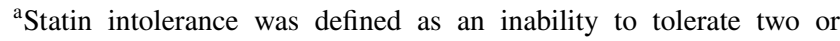
more statins (one statin at the lowest daily starting dose [i.e., rosuvastatin $5 \mathrm{mg}$, atorvastatin $10 \mathrm{mg}$, simvastatin $10 \mathrm{mg}$, lovastatin $20 \mathrm{mg}$, pravastatin $40 \mathrm{mg}$, fluvastatin $40 \mathrm{mg}$, or pitavastatin $2 \mathrm{mg}$ ], and another statin at any dose) owing to unexplained skeletal muscle-related symptoms, other than as a result of strain or trauma, that began or increased during statin treatment and resolved with statin discontinuation

${ }^{\mathrm{b}}$ Partial statin intolerance was defined as an inability to tolerate sufficient statin dose to reach treatment target

12 weeks (week 12; visit 4) and 24 weeks of treatment (week 24; visit 5). Optional documentation of data was possible at weeks 2 (visit 2) and 4 (visit 3 ) if a routine visit was scheduled independently of study participation.

The relative change in LDL-C at week 24 in comparison to baseline was calculated as follows: $100 \times$ (week 24 LDL-C - baseline LDL-C)/baseline LDL-C. The non-HDL-C percentage change from baseline to week 24 was calculated post-hoc as follows: total cholesterol-HDL-C. Physicians provided $\mathrm{HbA}_{1 \mathrm{c}}$ levels as percentage values. 
Safety was assessed by monitoring adverse events (AEs), serious AEs, and fatal events. Adverse events were defined as AEs reported from the time the physician obtained the patient's informed consent until the end of the study period plus 7 days. Adverse events were reported within 24 hours (or on the next working day) via fax or e-mail to the designated clinical research organization. Furthermore, details on AEs were entered in the case report form. Adverse events of special interest included pregnancy of a participating female patient or the partner of a participating male patient, symptomatic overdose, and an increase in alanine aminotransferase; these had to be reported instantaneously (i.e., within 24 hours). The safety analysis set included all patients who received one or more doses of alirocumab within this study.

\subsection{Statistical Analysis}

Quantitative data of continuous variables (e.g., age) were summarized by mean ( \pm standard deviation). The interquartile range (Q1: 25th percentile; Q3: 75th percentile) was used when presenting results for skewed distributions. Qualitative data of categorical variables (e.g., sex) were presented by means of (absolute and relative) frequency distributions. In cases of missing data, two methods were followed for calculation of percentages: the first method considered missing data as a separate group; the second method was based on the valid data per parameter. Two-sided $95 \%$ confidence intervals for the mean were based on a normal approximation for quantitative variables, and on exact methods for binomial proportions (Clopper-Pearson type intervals) for dichotomous variables.

A pre-specified modified ITT analysis was also performed for the percentage change in LDL-C from baseline to week 24 and key secondary endpoints, including all patients included in the ITT analysis who had an LDL-C value at baseline and at week 24. Consistency of treatment effect across subgroups was assessed by providing interaction $p$ values. All statistical analyses were carried out using SAS $^{\circledR}$ (Version 9.4).

\section{Results}

The PEARL study cohort consisted of 619 patients (Fig. 1 of the ESM). All patients received at least one dose of alirocumab within this study (safety population). The ITT population included 612 patients overall. The modified ITT population comprised 491 patients for whom the primary endpoint could be evaluated.

At baseline (prior to the first alirocumab dose), 50.8\% of patients had FH and $27.6 \%$ had DM (Table 1). Most patients $(90.2 \%)$ had a history of cardiovascular disease, including $72.4 \%$ with a history of CHD and $33.0 \%$ who were
post-ACS. In patients with CHD, peripheral arterial disease was the most common comorbidity (Fig. 1). Before the start of alirocumab therapy, total statin intolerance was reported by $45.3 \%$ of patients and partial statin intolerance by $27.6 \%$. The number of statins according to statin intolerance status prior to the study start is presented in Table 2 of the ESM. Baseline mean LDL-C level was $4.7 \mathrm{mmol} / \mathrm{L}$ (180.5 mg/dL), despite ongoing non-alirocumab LLT (Table 1).

\subsection{Efficacy Analysis}

The initial alirocumab dose was $75 \mathrm{mg} \mathrm{Q} 2 \mathrm{~W}$ in $72.9 \%$ of patients and $150 \mathrm{mg} \mathrm{Q} 2 \mathrm{~W}$ in $24.5 \%$ (unknown dosing regimen: $2.6 \%$; Table 3 of the ESM). During the study, $19.6 \%$ of patients received an alirocumab dose increase from $75 \mathrm{mg}$ to $150 \mathrm{mg} \mathrm{Q} 2 \mathrm{~W}$, and in $1.6 \%$ of patients the dose was decreased from $150 \mathrm{mg}$ to $75 \mathrm{mg} \mathrm{Q} 2 \mathrm{~W}$ (discontinuation of treatment: $12.3 \%$; missing: $2.8 \%$ ). In addition, $43.6 \%$ of patients continued taking alirocumab $75 \mathrm{mg} \mathrm{Q} 2 \mathrm{~W}$ and $20.1 \%$ continued taking alirocumab $150 \mathrm{mg} \mathrm{Q} 2 \mathrm{~W}$ throughout the study. A detailed overview of the alirocumab starting dose (week 0) and treatment status at weeks 12 and 24 is presented in Table 4 of the ESM.

The mean percentage change from baseline to week 24 in LDL-C was $-48.6 \%$ ( $p<0.0001$ vs. baseline). These LDL-C reductions allowed $48.5 \%(n=297)$ of patients to achieve LDL-C levels of $\leq 1.8$ or $\leq 2.6 \mathrm{mmol} / \mathrm{L}$ ( $\leq 70$ or $\leq 100 \mathrm{mg} / \mathrm{dL}$; depending on cardiovascular risk) and $55.6 \%$ to achieve their individual LDL-C target levels (defined by their treating physician) at week 24. Alirocumab maintained consistent LDL-C reductions throughout the study, with reductions being observed from week 2 and remaining largely constant from weeks 4 to 24 (Fig. 2). At week 24, mean (standard deviation) LDL-C was 2.3 (1.2) $\mathrm{mmol} / \mathrm{L}$ (89.8 [46.0] mg/dL; Table 2). The mean absolute and percentage changes from

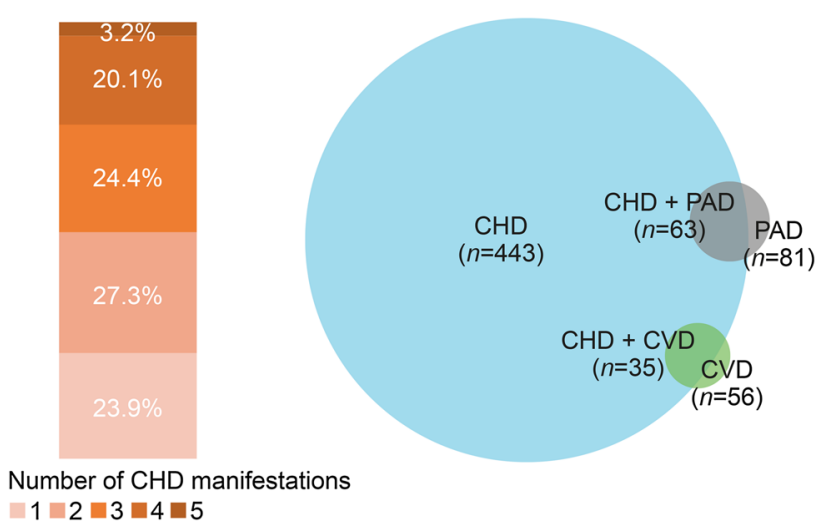

Fig. 1 Patients with coronary heart disease (CHD) according to the number of manifestations and comorbidities (intention-to-treat analysis). $C V D$ cerebrovascular disease, $P A D$ peripheral arterial disease 
Table 2 Effect of alirocumab on low-density lipoprotein cholesterol (LDL-C), secondary lipid parameters, and LDL-C target levels at baseline and week 24 (intention-to-treat [ITT] analysis)

\begin{tabular}{|c|c|c|c|c|c|}
\hline & $\begin{array}{l}\text { Baseline, mean (SD), } \\
\mathrm{mmol} / \mathrm{L}[\mathrm{mg} / \mathrm{dL}]\end{array}$ & $\begin{array}{l}\text { Week } 24, \text { mean }(\mathrm{SD}) \text {, } \\
\mathrm{mmol} / \mathrm{L}[\mathrm{mg} / \mathrm{dL}]\end{array}$ & $\begin{array}{l}\text { Mean absolute change } \\
\text { from baseline }{ }^{\mathrm{a}}, \text { mean }(\mathrm{SD}) \text {, } \\
\mathrm{mmol} / \mathrm{L}[\mathrm{mg} / \mathrm{dL}]\end{array}$ & $\begin{array}{l}\text { Change from baseline } \\
\text { to week } 24^{\mathrm{a}}, \mathrm{LS} \text { mean, } \\
\%\end{array}$ & $p$ value \\
\hline LDL-C & $4.7(1.6)[180.5(60.7)]$ & $2.3(1.2)[89.8(46.0)]$ & $-2.3(1.4)[-90.3(53.8)]$ & -48.6 & $<0.0001$ \\
\hline Non-HDL-C & $5.5(1.9)[213.9(72.2)]$ & $2.9(1.4)[113.6(52.8)]$ & $-2.6(1.7)[-101.9(65.5)]$ & -45.8 & $<0.0001$ \\
\hline HDL-C & $1.3(0.5)[50.8(19.7)]$ & $1.4(0.4)[52.6(16.6)]$ & $0.1(0.3)[2.0(13.1)]$ & 7.1 & $<0.0001$ \\
\hline Total cholesterol & $6.9(2.0)[266.6(76.1)]$ & $4.3(1.4)[167.2(55.2)]$ & $-2.6(1.7)[-99.5(64.6)]$ & -35.7 & $<0.0001$ \\
\hline TGs & $2.9(4.3)[256.8(377.9)]$ & $2.1(2.2)[186.9(191.8)]$ & $-0.9(3.5)[-76.6(305.7)]$ & -11.0 & $<0.0001$ \\
\hline Median (Q1:Q3) & $\begin{array}{l}2.0(1.5: 3.1) \\
\quad[178.0(129.0: 268.0)]\end{array}$ & $\begin{array}{l}1.6(1.2: 2.5 \\
\quad[144.0(106.0: 218.0)]\end{array}$ & $\begin{array}{l}-0.3(-1.0: 0.1 \\
\quad[-29.0(-85.0: 9.0)]\end{array}$ & & \\
\hline
\end{tabular}

$H D L-C$ high-density lipoprotein cholesterol, $L S$ least squares, $S D$ standard deviation, $T G$ triglyceride

${ }^{a}$ Data presented for the modified ITT population

baseline to week 24 in LDL-C, non-HDL-C, HDL-C, total cholesterol, and TGs are presented in Table 2. Significant reductions in non-HDL-C, total cholesterol, and TGs, and

Table 3 Alirocumab efficacy on low-density lipoprotein cholesterol (LDL-C) levels according to patient subgroups (intention-to-treat [ITT] analysis)

\begin{tabular}{|c|c|c|c|c|c|c|}
\hline & $n^{\mathrm{a}}$ & $\begin{array}{l}\text { Baseline } \\
\text { mean }(\mathrm{SD}), \mathrm{mmol} / \mathrm{L} \\
{[\mathrm{mg} / \mathrm{dL}]}\end{array}$ & $n^{\mathrm{a}}$ & $\begin{array}{l}\text { Week } 24 \\
\text { mean }(\mathrm{SD}), \mathrm{mmol} / \mathrm{L} \\
{[\mathrm{mg} / \mathrm{dL}]}\end{array}$ & $\begin{array}{l}\text { Percentage change from } \\
\text { baseline to week } 24,{ }^{b} \\
\text { LS mean, } \%\end{array}$ & $\begin{array}{l}\text { Interaction } \\
p \text { value }\end{array}$ \\
\hline $\mathrm{FH}$ & 299 & $5.1(1.7)[195.8(66.3)]$ & 254 & 2.5 (1.4) [97.4 (53.7)] & -49.1 & 0.6500 \\
\hline Non-FH & 34 & $4.1(1.1)[157.5(41.5)]$ & 28 & $2.1(0.9)[79.3(33.2)]$ & -51.4 & \\
\hline Unknown FH status & 261 & $4.3(1.3)[166.0(50.9)]$ & 224 & $2.1(0.9)[82.6(35.2)]$ & -47.7 & \\
\hline Type 1 DM & 10 & $3.7(0.9)[144.0(35.7)]$ & 7 & $1.9(0.5)$ [73.9 (19.2)] & -48.4 & 0.7930 \\
\hline Type 2 DM & 150 & 4.5 (1.6) [175.4 (63.3)] & 130 & $2.3(1.1)[87.2(42.9)]$ & -47.4 & \\
\hline Non-DM & 424 & $4.8(1.5)[184.4(59.6)]$ & 363 & $2.4(1.2)[91.6(47.5)]$ & -49.0 & \\
\hline $\mathrm{CHD}$ & 434 & $4.5(1.5)[172.3(57.7)]$ & 384 & $2.2(1.0)[83.7(40.0)]$ & -50.0 & 0.1542 \\
\hline Non-CHD & 82 & $5.0(1.4)[191.2(54.4)]$ & 70 & $2.6(1.2)[100.0(45.2)]$ & -45.6 & \\
\hline Post-ACS & 194 & $4.3(1.3)[166.0(50.8)]$ & 186 & $2.1(1.0)$ [80.5 (39.1)] & -50.4 & 0.7891 \\
\hline No ACS & 227 & $4.8(1.5)[184.9(57.9)]$ & 191 & $2.4(1.1)[91.1(43.0)]$ & -49.8 & \\
\hline Male & 383 & $4.4(1.5)[171.2(56.7)]$ & 332 & $2.2(1.2)[84.1(44.7)]$ & -49.2 & 0.3595 \\
\hline Female & 207 & $5.1(1.7)[198.8(63.9)]$ & 172 & $2.6(1.2)[101.3(46.5)]$ & -47.2 & \\
\hline $\begin{array}{l}\text { Baseline LDL-C }<3.4 \mathrm{mmol} / \mathrm{L} \\
(<130 \mathrm{mg} / \mathrm{dL})\end{array}$ & 112 & $2.7(0.5)[102.9(20.8)]$ & 92 & $1.7(0.9)[64.2(35.1)]$ & -38.1 & $<0.0001$ \\
\hline $\begin{array}{l}\text { Baseline LDL-C } \geq 3.4 \text { to } \\
\leq 4.9 \mathrm{mmol} / \mathrm{L}(\geq 130 \\
\text { to } \leq 190 \mathrm{mg} / \mathrm{dL})\end{array}$ & 250 & $4.2(0.4)[162.0(17.1)]$ & 205 & $2.1(0.8)[80.3(31.1)]$ & -50.1 & \\
\hline $\begin{array}{l}\text { Baseline LDL-C }>4.9 \mathrm{mmol} / \mathrm{L} \\
(>190 \mathrm{mg} / \mathrm{dL})\end{array}$ & 232 & $6.2(1.2)[237.9(48.3)]$ & 194 & $2.9(1.4)[113.0(53.0)]$ & -52.0 & \\
\hline Total statin intolerance & 267 & $5.0(1.6)[193.1(60.1)]$ & 227 & $2.6(1.2)[99.3(44.7)]$ & -47.6 & 0.1302 \\
\hline Partial statin intolerance & 150 & $4.3(1.4)[167.8(54.5)]$ & 132 & $2.0(1.0)[77.2(38.1)]$ & -52.2 & \\
\hline No statin intolerance & 154 & $4.4(1.6)[170.5(63.7)]$ & 131 & $2.2(1.3)[85.6(50.8)]$ & -47.1 & \\
\hline
\end{tabular}

$\overline{A C S}$ acute coronary syndrome, $C H D$ coronary heart disease, $D M$ diabetes mellitus, $F H$ familial hypercholesterolemia, $L S$ least squares, $S D$ standard deviation

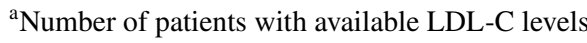

${ }^{\mathrm{b}}$ Data presented for the modified ITT population 
an increase in HDL-C, were observed from baseline to week 24 (all $p<0.0001)$.

The efficacy of alirocumab was consistent in patient subgroups according to FH, DM, CHD, ACS, baseline LDL-C, and statin intolerance, with least-squares mean percentage reductions being at least $38.1 \%$ from baseline to week 24 in each subgroup (Table 3). Similar results were observed in the modified ITT patient population (Table 5 of the ESM).

\subsection{Safety Analysis}

Adverse events were reported in $10.3 \%$ of patients and the most common AEs were myalgia (7.3\% of total AEs [11/151]), dyspnea (3.3\% of total AEs [5/151]), and fatigue (3.3\% of total AEs [5/151]; Table 4). No AEs of special interest were reported. A total of 37 patients (6.0\%) prematurely discontinued the treatment following at least one AE. The most often reported AEs that led to treatment discontinuation according to reported AEs $(n=79)$ were LDL-C increased (nine events, $11.4 \%$ of total AEs events [9/79] that resulted in treatment discontinuation), myalgia (six events, 7.6\% [6/79]), and dyspnea (five events, 6.3\% [5/79]). One death was reported. The death of the patient was reported

Table 4 Safety summary (safety analysis)

\begin{tabular}{ll}
\hline$n(\%)$ & Alirocumab \\
\hline$n$ & 619 \\
Any AE & $64(10.3)$ \\
SAE & $9(1.5)$ \\
AE leading to death & $1(0.2)^{\mathrm{a}}$ \\
AE leading to permanent treatment discontinuation ${ }^{\mathrm{b}}$ & $37(6.0)$ \\
Any reported AE occurring $\geq 2 \%$ as a proportion of all reported \\
AEs $(n=151)$ & \\
Myalgia & $11(7.3)$ \\
Dyspnea & $5(3.3)$ \\
Fatigue & $5(3.3)$ \\
Blood creatine phosphokinase increased & $4(2.6)$ \\
Diarrhea & $4(2.6)$ \\
Nausea & $4(2.6)$ \\
Pruritus & $4(2.6)$ \\
Rash & $4(2.6)$ \\
Arthralgia & $3(2.0)$ \\
Erythema & $3(2.0)$ \\
Headache & $3(2.0)$ \\
LDL increased & $3(2.0)$ \\
Pain in extremity & $3(2.0)$ \\
\hline
\end{tabular}

$A E$ adverse event, $L D L$ low-density lipoprotein, $S A E$ serious adverse event

${ }^{\mathrm{a}}$ The death was reported as not related to alirocumab treatment by a physician

${ }^{\mathrm{b}}$ Including AEs that required action

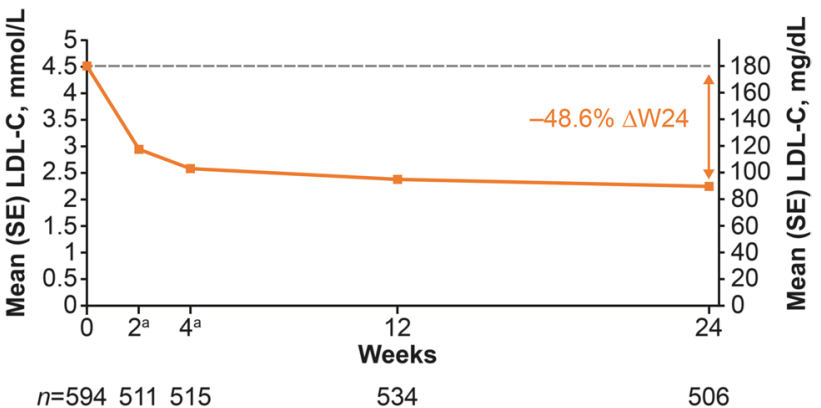

Fig. 2 Mean (standard error [SE]) low-density lipoprotein cholesterol

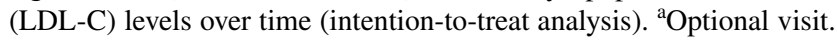
$\Delta W 24$ percent change from baseline to week 24

as not related to alirocumab treatment by the physician, and the patient's malignant disease was specified as the cause of death.

In the overall PEARL study population, mean (standard deviation) $\mathrm{HbA}_{1 \mathrm{c}}$ levels were similar at baseline (6.5\% [1.2]) and at week 24 (6.1\% [1.0]). In patients with $\mathrm{DM}, \mathrm{HbA}_{1 \mathrm{c}}$ levels were 6.9\% (1.2) at baseline and 6.7\% (1.0) at week 24.

\section{Discussion}

In a real-world setting, the PEARL study data showed that alirocumab was mostly used in patients with high cardiovascular risk who had reported previous or current statin intolerance, defined as reported intolerance to two or more statins. In total, $72.4 \%$ of patients had a history of CHD, and $45.3 \%$ and $27.6 \%$ reported total and partial statin intolerance, respectively. In PEARL, 50.8\% of enrolled patients experienced FH.

At week 24, alirocumab reduced LDL-C levels from baseline by $48.6 \%$. In the double-blind ODYSSEY ALTERNATIVE study, a LDL-C reduction of $45.0 \%$ from baseline to week 24 was observed in statin-intolerant patients treated with alirocumab $75 \mathrm{mg}$ Q2W (with possible dose increase to $150 \mathrm{mg}$ Q2W) [10]. In ODYSSEY OLE, similar results were observed up to week 96 in alirocumab-treated patients with $\mathrm{HeFH}$ (47.9\% LDL-C reduction from baseline) [22]. The observed alirocumab efficacy was also consistent with that observed in a pooled analysis including 4629 patients with high cardiovascular risk from eight ODYSSEY Phase III studies, in particular those with the alirocumab 75-mg Q2W dosing regimen with a possible dose adjustment to $150 \mathrm{mg}$ Q2W at week 12 (44.5\% LDL-C reduction) [9]. These data are also similar to an alirocumab expanded use program reporting a 55.1\% LDL-C reduction from baseline to week 24 in patients with $\mathrm{HeFH}$ and/or CHD in the USA who received alirocumab $150 \mathrm{mg}$ Q2W [23], an audit study that reported a 49\% LDL-C reduction in 105 PCSK9 
inhibitor-treated patients receiving maximally tolerated statin therapy in the UK [24] and a cohort of 238 patients who received either alirocumab or evolocumab in routine outpatient care in the Netherlands (55.0\% LDL-C reduction from baseline) [18].

Owing to the required treatment according to the summary of product characteristics as part of the study inclusion criteria for PEARL, nearly all patients should have received maximally tolerated statin treatment prior to the start of alirocumab therapy, including patients with total $(45.3 \%)$ and partial statin intolerance (27.6\%). During PEARL, 43.6\% of patients continued taking alirocumab $75 \mathrm{mg}$ Q2W without requiring a dose increase. Similarly, in the OLE study, among patients receiving the alirocumab starting dose of $75 \mathrm{mg}$ Q2W, 56.7\% were maintained on that dose for over 3 years based on a physician's clinical judgment and the patient's LDL-C levels from week 8 [22].

Overall, $48.5 \%$ of patients reached LDL-C levels of $\leq 1.8$ or $\leq 2.6 \mathrm{mmol} / \mathrm{L}$ ( $\leq 70$ or $\leq 100 \mathrm{mg} / \mathrm{dL}$; depending on cardiovascular risk) at week 24 . Similar results were observed at week 24 in the ALTERNATIVE study (41.9\%) and at week 96 in the OLE study $(55.3 \%)[10,22]$. Several guidelines recommend treatment goals of LDL-C $\leq 1.8$ or $\leq 2.6 \mathrm{mmol} / \mathrm{L}$ $(\leq 70$ or $\leq 100 \mathrm{mg} / \mathrm{dL})$ in patients with high or very high cardiovascular risk, including those with peripheral arterial disease and/or DM [4, 6, 25]. These data might indicate a better treatment adherence than has been previously reported by an audit study (July 2016 to December 2016) from the UK showing $30 \%$ of patients with mean baseline LDL-C $5.3 \mathrm{mmol} / \mathrm{L}(203.0 \mathrm{mg} / \mathrm{dL})$ achieving LDL-C $<2.5 \mathrm{mmol} / \mathrm{L}$ $(<96.7 \mathrm{mg} / \mathrm{dL})$, despite a similar study discontinuation rate (15\%) vs. PEARL $(4.7 \mathrm{mmol} / \mathrm{L}[180.5 \mathrm{mg} / \mathrm{dL}]$ and $13.4 \%$, respectively) [24].

The PEARL study showed that alirocumab may be a suitable treatment option for patients with high cardiovascular risk and those with $\mathrm{HeFH}$ or DM. Consistent with a pooled analysis of five placebo-controlled studies, no difference in the LDL-C reduction was observed in alirocumab-treated patients with and without DM [26]. Furthermore, in insulintreated patients at high cardiovascular risk with type 1 and type $2 \mathrm{DM}$, alirocumab reduced LDL-C levels from baseline at week 24 by $47.8 \%$ and $49.0 \%$, respectively [27]. Similar reductions were observed in patients with type $2 \mathrm{DM}$ and mixed dyslipidemia [28]. Consistent with alirocumabtreated individuals with and without DM enrolled for clinical studies, no increase in $\mathrm{HbA}_{1 \mathrm{C}}$ levels was observed [27-29]. In our study, we observed similar LDL-C reductions from baseline to week 24 in patients with and without FH (49.1\% and $51.4 \%$, respectively). These data are again consistent with a previously reported $48.8 \%$ reduction from a pool of two ODYSSEY studies with the 75-mg Q2W dosing regimen (with a possible dose adjustment to $150 \mathrm{mg}$ Q2W) [30]. A registry study from the Netherlands reported an LDL-C reduction of 55\% in patients with $\mathrm{HeFH}$ who received either alirocumab or evolocumab in addition to maximally tolerated LLT [31].

Alirocumab was generally well tolerated, with $10.3 \%$ of patients reporting AEs. Considering that in PEARL most of the patients were defined to be statin intolerant (either full or partial), this is a low rate of AEs compared with reported treatment-emergent AEs in the double-blind ALTERNATIVE study [10]. In a pooled analysis from 14 alirocumab clinical studies ( $n=3340$ alirocumab-treated patients), a similar rate of treatment-emergent AEs was reported compared with patients in the placebo $(n=1276)$ and ezetimibe $(n=618)$ groups, with a higher incidence of local injection-site reactions [32]. Similar to the ALTERNATIVE study, myalgia was the most common AE in PEARL [10]. Direct comparisons of reported AEs between ODYSSEY studies and the PEARL study should be performed with caution, as ODYSSEY studies assessed treatment-emergent AEs according to the number of patients whereas PEARL reported AEs according to the number of total AEs [10, 15, 22]. In PEARL, 151 AEs were reported throughout, including 11 reported events of myalgia (7.3\% of total number of $\mathrm{AE}$ events). Enrolled patients were able to report a specific AE more than once throughout the study, i.e., myalgia might have been reported by fewer than 11 patients. In total, three events (2.0\%) of increased LDL were reported. Most likely, this reflects a change in the concomitant LLTs; however, no further information was available about the LLT status for patients reporting this $\mathrm{AE}$.

To our knowledge, this is the largest study to date showing the efficacy and safety of a PCSK9 inhibitor in a realworld setting up to 24 weeks. A limitation of this study is the unknown status of LLT therapy for enrolled patients because of an unclear patient questionnaire that resulted in inconsistencies specifically and exclusively in the respective data. Other limitations could include the 24-week study duration for the long-term efficacy and safety assessment of alirocumab in a real-world setting as well as the confirmation of FH status that was based on an algorithm and criteria used in Germany [33]. Furthermore, the open-label treatment of alirocumab might have introduced a bias by study participants and physicians [34].

\section{Conclusions}

The PEARL study suggests that, in a real-world setting, alirocumab is a beneficial LLT to further reduce LDL-C levels in patients at cardiovascular risk on maximally tolerated statin treatment with/without other non-alirocumab LLT.

Acknowledgements The following people reviewed and provided editorial comments on the manuscript: Gloria Garcia-Palacios, Michael 
Howard, Nicole Parsons, and Robert Wright (Sanofi), and Carol Hudson, Marc Israel, and Robert Pordy (Regeneron Pharmaceuticals, Inc.). Medical writing support was provided by Susanne Ulm, PhD, of Prime (Knutsford, UK), and was funded by Sanofi and Regeneron Pharmaceuticals, Inc. according to Good Publication Practice guidelines. The sponsor was involved in the study design, collection, and analysis and interpretation of data, as well as data checking of information provided in the manuscript.

\section{Compliance with Ethical Standards}

Funding This study was sponsored by Sanofi-Aventis Deutschland and funded by Sanofi and Regeneron Pharmaceuticals, Inc.

Conflict of interest Klaus G. Parhofer has received research support from Regeneron Pharmaceuticals Inc. and Sanofi, has received consulting fees or honoraria from Aegerion, Akcea, Amgen, Ionis, Regeneron Pharmaceuticals, Inc., and Sanofi, and has received fees for participation in review activities from Boehringer-Ingelheim. Berndt von Stritzky, Nicole Pietschmann, Cornelia Dorn, and W. Dieter Paar are employees of and stockholders in Sanofi.

Author contributions All authors contributed to the concept or design of the study, data analysis, or interpretation of data, and critically reviewed and edited the manuscript. All authors approved the final version.

Open Access This article is distributed under the terms of the Creative Commons Attribution-NonCommercial 4.0 International License (http://creativecommons.org/licenses/by-nc/4.0/), which permits any noncommercial use, distribution, and reproduction in any medium, provided you give appropriate credit to the original author(s) and the source, provide a link to the Creative Commons license, and indicate if changes were made.

\section{References}

1. Piepoli MF, Hoes AW, Agewall S, Albus C, Brotons C, Catapano AL, et al. 2016 European guidelines on cardiovascular disease prevention in clinical practice: the Sixth Joint Task Force of the European Society of Cardiology and Other Societies on Cardiovascular Disease Prevention in Clinical Practice (constituted by representatives of 10 societies and by invited experts) developed with the special contribution of the European Association for Cardiovascular Prevention \& Rehabilitation (EACPR). Eur Heart J. 2016;37(29):2315-81.

2. Lloyd-Jones DM, Morris PB, Ballantyne CM, Birtcher KK, Daly DD Jr, DePalma SM, et al. 2017 focused update of the 2016 ACC expert consensus decision pathway on the role of non-statin therapies for LDL-cholesterol lowering in the management of atherosclerotic cardiovascular disease risk: a report of the American College of Cardiology Task Force on Expert Consensus Decision Pathways. J Am Coll Cardiol. 2017;70(14):1785-822.

3. Ibanez B, James S, Agewall S, Antunes MJ, Bucciarelli-Ducci C, Bueno H, et al. 2017 ESC guidelines for the management of acute myocardial infarction in patients presenting with ST-segment elevation. Kardiol Pol. 2018;76(2):229-313.

4. American Association of Clinical Endocrinologists, American College of Endocrinology. AACE/ACE comprehensive type 2 diabetes management algorithm 2017. Endocr Pract. 2017. https ://doi.org/10.4158/ep161682.cs.
5. American Diabetes Association. Standards of medical care in diabetes: 2018. Diabetes Care. 2018;41(Suppl. 1):S1-159.

6. Landmesser U, Chapman MJ, Stock JK, Amarenco P, Belch JJF, Boren J, et al. 2017 Update of ESC/EAS Task Force on practical clinical guidance for proprotein convertase subtilisin/kexin type 9 inhibition in patients with atherosclerotic cardiovascular disease or in familial hypercholesterolaemia. Eur Heart J. 2018;39(14):1131-43.

7. Sanofi-Aventis U.S. LLC. Praluent prescribing information (US). 2015. http://products.sanofi.us/praluent/praluent.pdf. [Accessed 8 Aug 2017].

8. Sanofi-Aventis Groupe. Praluent summary of product characteristics. https://www.medicines.org.uk/emc/medicine/30956. Accessed 2 Jul 2018.

9. Farnier M, Gaudet D, Valcheva V, Minini P, Miller K, Cariou B. Efficacy of alirocumab in high cardiovascular risk populations with or without heterozygous familial hypercholesterolemia: pooled analysis of eight ODYSSEY Phase 3 clinical program trials. Int J Cardiol. 2016;223:750-7.

10. Moriarty PM, Thompson PD, Cannon CP, Guyton JR, Bergeron $\mathrm{J}$, Zieve FJ, et al. Efficacy and safety of alirocumab vs ezetimibe in statin-intolerant patients, with a statin rechallenge arm: the ODYSSEY ALTERNATIVE randomized trial. J Clin Lipidol. 2015;9(6):758-69.

11. Roth EM, Taskinen MR, Ginsberg HN, Kastelein JJ, Colhoun HM, Robinson JG, et al. Monotherapy with the PCSK9 inhibitor alirocumab versus ezetimibe in patients with hypercholesterolemia: results of a 24 week, double-blind, randomized phase 3 trial. Int J Cardiol. 2014;176(1):55-61.

12. Roth EM, Moriarty PM, Bergeron J, Langslet G, Manvelian G, Zhao J, et al. A phase III randomized trial evaluating alirocumab $300 \mathrm{mg}$ every 4 weeks as monotherapy or add-on to statin: ODYSSEY CHOICE I. Atherosclerosis. 2016;254:254-62.

13. Moriarty PM, Parhofer KG, Babirak SP, Cornier MA, Duell PB, Hohenstein B, et al. Alirocumab in patients with heterozygous familial hypercholesterolaemia undergoing lipoprotein apheresis: the ODYSSEY ESCAPE trial. Eur Heart J. 2016;37(48):3588-95.

14. Stroes E, Guyton JR, Lepor N, Civeira F, Gaudet D, Watts $\mathrm{GF}$, et al. Efficacy and safety of alirocumab $150 \mathrm{mg}$ every 4 weeks in patients with hypercholesterolemia not on statin therapy: the ODYSSEY CHOICE II study. J Am Heart Assoc. 2016;5(9):e003421.

15. Schwartz GG, Steg PG, Szarek M, Bhatt DL, Bittner VA, Diaz R, et al. Alirocumab and cardiovascular outcomes after acute coronary syndrome. N Engl J Med. 2018;379:2097-107.

16. Sabatine MS, Giugliano RP, Keech AC, Honarpour N, Wiviott SD, Murphy SA, et al. Evolocumab and clinical outcomes in patients with cardiovascular disease. N Engl J Med. 2017;376(18):1713-22.

17. Gemainsamer Bundesausschuss. Drug directive/appendix III: alirocumab. 2016. https://www.g-ba.de/informationen/beschluess e/2676/. Accessed 26 Jun 2018.

18. Stoekenbroek RM, Hartgers ML, Rutte R, de Wijer DD, Stroes ESG, Hovingh GK. PCSK9 inhibitors in clinical practice: delivering on the promise? Atherosclerosis. 2018;270:205-10.

19. Hollstein T, Grenkowitz T, Spira D, Scharnagl H, März W, Steinhagen-Thiessen E, et al. Abstract 16464: proprotein convertase subtilisin/kexin type 9 antibodies (pcsk9-i) treatment of cardiovascular high risk patients in clinical practice: new data regarding safety and effectivity. Circulation. 2017;136:A16464.

20. Waldmann E, Bamberger C, Parhofer KG. Statin intolerance is the main indication for PCSK9 inhibition in clinical practice. Atherosclerosis. 2017;263:e80.

21. Sanofi-Aventis Deutschland GmbH. Prospektive Nicht-Interventionelle Studie zur Erfassung der Wirksamkeit und Verträglichkeit des PCSK9-Inhibitors Alirocumab (Praluent)_PEARL. 2017. 
https://www.pei.de/SharedDocs/awb/nis-0301-0400/0320.html. Accessed 21 Jun 2018.

22. Farnier M, Hovingh GK, Langslet G, Dufour R, Baccara-Dinet MT, Din-Bell C, et al. Long-term safety and efficacy of alirocuamb in patients with heterozygous familial hypercholesterolemia: an open-label extension of the ODYSSEY program. Atherosclerosis. 2018;278:307-14.

23. Glueck CJ, Brown A, Goldberg AC, McKenney JM, Kantaros L, Stewart J, et al. Alirocumab in high-risk patients: observations from the open-label expanded use program. J Clin Lipidol. 2018;12(3):662-8.

24. Kohli M, Patel K, MacMahon Z, Ramachandran R, Crook MA, Reynolds TM, et al. Pro-protein subtilisin kexin-9 (PCSK9) inhibition in practice: lipid clinic experience in 2 contrasting UK centres. Int J Clin Pract. 2017;71(11):e13032.

25. Aboyans V, Ricco JB, Bartelink MEL, Bjorck M, Brodmann M, Cohnert T, et al. 2017 ESC guidelines on the diagnosis and treatment of peripheral arterial diseases, in collaboration with the European Society for Vascular Surgery (ESVS). Rev Esp Cardiol (English ed). 2018;71(2):111.

26. Ginsberg HN, Farnier M, Robinson JG, Cannon CP, Sattar N, Baccara-Dinet MT, et al. Efficacy and safety of alirocumab in individuals with diabetes mellitus: pooled analyses from five placebocontrolled phase 3 studies. Diabetes Ther. 2018;9(3):1317-34.

27. Leiter LA, Cariou B, Muller-Wieland D, Colhoun HM, Del Prato $\mathrm{S}$, Tinahones FJ, et al. Efficacy and safety of alirocumab in insulin-treated individuals with type 1 or type 2 diabetes and high cardiovascular risk: the ODYSSEY DM-INSULIN randomized trial. Diabetes Obes Metab. 2017;19(12):1781-92.

28. Ray KK, Leiter LA, Muller-Wieland D, Cariou B, Colhoun HM, Henry RR, et al. Alirocumab vs usual lipid-lowering care as addon to statin therapy in individuals with type 2 diabetes and mixed dyslipidaemia: the ODYSSEY DM-DYSLIPIDEMIA randomized trial. Diabetes Obes Metab. 2018;20(6):1479-89.

29. Colhoun HM, Ginsberg HN, Robinson JG, Leiter LA, MullerWieland D, Henry RR, et al. No effect of PCSK9 inhibitor alirocumab on the incidence of diabetes in a pooled analysis from 10 ODYSSEY Phase 3 studies. Eur Heart J. 2016;37(39):2981-9.

30. Kastelein JJ, Ginsberg HN, Langslet G, Hovingh GK, Ceska R, Dufour R, et al. ODYSSEY FH I and FH II: 78 week results with alirocumab treatment in 735 patients with heterozygous familial hypercholesterolaemia. Eur Heart J. 2015;36(43):2996-3003.

31. Galema-Boers AMH, Lenzen MJ, Sijbrands EJ, Roeters van Lennep JE. Proprotein convertase subtilisin/kexin 9 inhibition in patients with familial hypercholesterolemia: initial clinical experience. J Clin Lipidol. 2017;11(3):674-81.

32. Jones PH, Bays HE, Chaudhari U, Pordy R, Lorenzato C, Miller $\mathrm{K}$, et al. Safety of alirocumab (a PCSK9 monoclonal antibody) from 14 randomized trials. Am J Cardiol. 2016;118(12):1805-11.

33. Klose G, Laufs U, Marz W, Windler E. Familial hypercholesterolemia: developments in diagnosis and treatment. Dtsch Arztebl Int. 2014;111(31-32):523-9.

34. Chan AW, Tetzlaff JM, Gotzsche PC, Altman DG, Mann H, Berlin JA, et al. SPIRIT 2013 explanation and elaboration: guidance for protocols of clinical trials. BMJ. 2013;346:e7586. 